\title{
A Coordination Mechanism with Fair Cost Allocation for Divergent Multi-Echelon Inventory Systems
}

\author{
Judith Timmer*
}

\begin{abstract}
This paper is concerned with the coordination of inventory control in divergent multiechelon inventory systems under periodic review and decentralized control. All the installations track echelon inventories. Under decentralized control the installations will decide upon replenishment policies that minimize their individual inventory costs. In general these policies do not coincide with the optimal policies of the system under centralized control. Hence, the total cost under decentralized control is larger than under centralized control.

To remove this cost inefficiency, a simple coordination mechanism is presented that is initiated by the most downstream installations. The upstream installation increases its base stock level while the downstream installation compensates the upstream one for increased costs and provides it with additional side payments. We show that this mechanism coordinates the system; the global optimal policy of the system is the unique Nash equilibrium of the corresponding strategic game. Furthermore, the mechanism results in a fair allocation of the costs; all installations enjoy cost savings.
\end{abstract}

Key Words: Inventory, coordination mechanism, fair allocation, multi-echelon, stochastic demand.

2000 AMS Subject classification: 90B05, 91A10, 90B50.

\section{Introduction}

In this paper we study the coordination of inventory control in divergent multi-echelon inventory systems under periodic review. The stockpoints in such a system track echelon inventories and their inventory is controlled by replenishment policies. Often, these stockpoints are managed independently, that is, the system is under decentralized control, and each decides upon a replenishment policy that minimizes its individual inventory costs. This way, the stockpoints neglect the external effects of their decisions upon the other parties in the system. This results in larger total costs compared to the system under centralized control and the decisions are not not globally optimal for the inventory system, that is, they do not coincide with the optimal decisions under centralized control.

${ }^{*}$ Stochastic Operations Research Group, Department of Applied Mathematics, University of Twente, P.O. Box 217, 7500 AE Enschede, The Netherlands. Phone: +31-53-4893419; fax: +31-53-4893069; email: j.b.timmer@utwente.nl 
To overcome this cost inefficiency, the ideal situation would be to consider the system under centralized control. Then a central planner minimizes the total cost of the system and instructs all the stockpoints about the decisions they should take to achieve this global minimum. However, in practice stockpoints in an inventory system are not willing to give up their independence just like that.

Alternatively, there is a need for coordination between the parties involved. This may be achieved by implementing a suitable coordination mechanism. While maintaining decentralized control, the goal of this mechanism is to change the incentives of the stockpoints in such a way that after this change, the new decisions of the stockpoints coincide with the global optimal decisions under centralized control. Then the inventory system is coordinated. Further, the coordination mechanism should result in a fair allocation of the costs, where fair means that all stockpoints should enjoy cost savings, compared to the initial situation under decentralized control. In this paper we introduce and analyse a mechanism that coordinates the inventory system and results in a fair allocation of the costs.

In the literature, inventory systems are studied extensively. We focus on divergent multiechelon inventory systems, also named distribution or production systems, under periodic review. In Diks and De Kok (1998) these systems are studied under centralized control. They consider a fixed lead time, stochastic demand, linear inventory costs, and penalty costs for backorders. Under a balance assumption, every stockpoint is controlled by a replenishment policy. Such a policy of a stockpoint consists of its base-stock level and allocation functions, which allocate its stock in case it is insufficient to fulfill all demand. The main result of the paper is that the optimal replenishment policy for each stockpoint can be determined by system decomposition, in which the system is decomposed into one-dimensional problems.

It is recognized in the literature that decentralized control leads to larger costs for divergent multi-echelon inventory systems. Several papers study coordination mechanisms to overcome this. Often, game theory is used as a tool to study these mechanisms; see for example Osborne and Rubinstein (1994), and Tijs (2003). Cachon (2001) studies a distribution system under continuous review. In this system, all firms pay inventory and backorder cost. A retailer is faced with Poisson-distributed demand and uses an $(r, q)$ ordering policy for replenishment of his stock. The supplier serves the retailers on a first-come-first-serve basis. It is shown that in such a system the competitive solution need not coincide with the global optimum. Three cooperation strategies are discussed, and two of these imply that the global optimal solution is a Nash equilibrium of the corresponding strategic game.

A two-echelon serial supply chain under decentralized control where each party minimizes its discounted costs, is studied in Lee and Whang (1999). The authors assume that all installations belong to a single organisation. Under a so-called performance measurement scheme, which is based on three basic properties, the installations minimize their discounted costs by selecting the global optimal outcome.

A three-stage supply chain involving suppliers, manufacturers and retailers is studied in Seliaman and Ahmad (2008). In this chain, production and inventory decisions are made by the suppliers and manufacturers. The goal is to coordinate the production and inventory decisions such that the total cost of the system is minimized. To achieve this goal, two coordination mechanisms are considered. In the first mechanism, all stages adopt the same cycle time. In the second mechanism, within a stage the same cycle time is adopted and this cycle time is an integer multiple of the cycle time used at the adjacent downstream stage. Numerical results show that this latter mechanism has lower costs than the first one.

Inventory systems under periodic review are studied in Zijm and Timmer (2008). The 
authors focus on serial and distribution inventoy systems under decentralized control. The distribution system is a two-stage inventory system in which it is assumed that all the retailers are identical. A coordination mechanism is introduced in which, among others, the retailers compensate the supplier for cost increases. It is shown that this mechanism results in the global optimum being the unique Nash equilibrium of the corresponding strategic game. Furthermore, this mechanism has lower costs for all the firms compared to the situation under decentralized control.

Recently, several excellent surveys on coordination in general supply chains have been published (Arshinder and Deshmukh, 2008; Leng and Zhu, 2009; Li and Wang, 2007). In the second survey, the authors remark that in most papers the mechanisms achieve coordination of the supply chain, however "very few publications considered the 'fair' allocation of extra profit or cost savings generated by the coordination" (Leng and Zhu, 2009, p. 602). Our paper can be added to this small list of publications since we consider both coordination and fair cost allocation.

More precisely, in this paper we study the multi-echelon inventory system in Diks and De Kok (1998) but now under decentralized control. To overcome the resulting cost inefficiency, compared to the system under centralized control, we introduce a simple coordination mechanism that will result in a fair allocation of the costs. This mechanism is initiated by the most downstream installations. They start negotiations with their upstream supplier. The upstream installation increases its base stock level while the downstream installation compensates the upstream one for the increase of costs and provides it with additional side payments. These monetary incentives result in new policies selected by the stockpoints that imply cost savings for all these installations. Hereafter, the upstream installations start similar negotiations with their supplier, and so on. Hence, in an $N$-echelon inventory system there will be $N-1$ rounds of negotiations. Thereafter the final results of the negotiations will be communicated to the downstream installations. This takes an additional $N-2$ rounds of communication. It is shown that this mechanism coordinates the supply chain, that is, the global optimal policy of the inventory system is the unique Nash equilibrium of the corresponding strategic game. Furthermore, the coordination mechanism results in a fair allocation of the costs; all installations agree upon the implementation of thise mechanism because it results in lower costs for each installation.

This paper contributes to the literature as follows. Our mechanism (i) coordinates the inventory system, and (ii) results in a fair allocation of the costs. Besides, our mechanism is an generalisation of the mechanism in Zijm and Timmer (2008) in two ways. First, the system under consideration allows for unequal holding and penalty costs among the installations, whereas all installations are identical in Zijm and Timmer (2008). Second, in this work we consider coordination of a system with at least three stages, which is considerably more complex than a system with just two stages as in Zijm and Timmer (2008) since now multiple rounds of negotiation and feedback are needed in the coordination mechanism.

The outline of this paper is as follows. In the next section we introduce our model. The inventory system under decentralized control is analyzed in section 3 . Thereafter, in section 4 we introduce and study our coordination mechanism. Section 5 concludes. All proofs may be found in the appendix. 


\section{Model}

In this section we introduce our model, which is based on the model in Diks and De Kok (1998).

We consider a single-item three-stage divergent inventory system, which is a system where each stockpoint has a unique supplier. Denote this unique supplier or preceding stockpoint of stockpoint $i$ by pre $(i)$. Note that we use the terms stockpoint and installation interchangeably. An echelon is a set of stockpoints starting from a certain installation and including all stockpoints downstream, that is, toward the end-stockpoints that try to meet customer demand. Denote by $e(i)$ the echelon starting from installation $i$. The echelon stock consists of all stock at the stockpoint plus all stock in transition to or on hand at any installation downstream minus eventual backlogs at the end-stockpoints. Finally, the echelon inventory position equals the echelon stock plus materials that are ordered but not yet delivered at the most upstream stockpoint in the echelon.

Periodically, each stockpoint bases its decision with regard to replenishment of stock on its echelon inventory position. It decides upon a so-called base stock level, that is, each period the stock is replenished to this desired level. The base stock level of stockpoint $i$ is denoted by $y_{i}$. Let $V_{i}$ be the set of all stockpoints that are directly supplied by $i$, then $y_{i} \geq \sum_{n \in V_{i}} y_{n}$ because the firms track echelon inventories. For ease of notation let $\Delta_{i}=y_{i}-\sum_{n \in V_{i}} y_{n}$ be the physical stock at stockpoint $i$.

The most upstream stockpoint in the system, stockpoint 1, orders to an outside supplier, who always has sufficient stock. The stockpoints use periodic review policies to control their inventories. Every period stockpoint $i$ places an order for replenishment of stock to its base stock level $y_{i}$. This order arrives after a lead time of $L_{i}$ periods. Now one of two cases will occur. If the inventory covers the demand for installation $i$ then this demand is fulfilled; any remaining stock is kept at stockpoint $i$ for future use. Otherwise, if the echelon stock $x$ of installation $i$ is insufficient to satisfy all demand of its successors then this stock will be rationed according to the allocation functions $z_{j}(x), j \in V_{i}$, that efficiently distribute the available stock: $\sum_{j \in V_{i}} z_{j}(x)=x$. We assume that this rationing always allocates nonnegative quantities; this is the so-called balance assumption. Furthermore, if the echelon stock happens to be sufficient then each installation receives its requested amount: $z_{j}\left(\sum_{n \in V_{j}} y_{n}\right)=y_{j}$.

Let $E$ denote the set of end-stockpoints and $M$ the set of the other (intermediate) stockpoints. For ease of notation we define by $U_{i}$ the set of stockpoints on the path from the outside supplier to stockpoint $i$. Notice that $U_{1}=\emptyset$. Also, a low level code (LLC) is assigned to every stockpoint. This code shows the level in the supply chain where the stockpoint operates, counted from the most downstream stockpoints. So an end-stockpoint $n \in E$ has $\operatorname{LLC}(n)=1$ and an intermediate stockpoint $i \in M$ has $\operatorname{LLC}(i)=1+\max _{j \in V_{i}} \operatorname{LLC}(j)$. Denote by $W_{i}$ the stockpoints with low level code $i$. Notice that $W_{1}=E$.

Without loss of generality we assume that customer demand only occurs in the endstockpoints. During a period, demand between end-stockpoints may be correlated but demands across periods are independent and identically distributed. The stochastic demand for stockpoint $i$ during a single period has cumulative density function $F_{i}$ and expected value $\mu_{i}$. By $F_{i}^{(L)}$ we denote the cumulative density function of the demand for $i$ during $L$ periods with expected value $L \mu_{i}$. Any demand that cannot be satisfied immediately will be backordered and it incurs a penalty cost of $p_{i}$ per unit backordered per period. Stockpoint $i$ adds $h_{i}$ to the value of a unit of stock. Hence, stockpoint $i$ pays a holding cost $h_{i}+\sum_{j \in U_{i}} h_{j}$ per unit of stock per period. 
A policy of an echelon prescribes for all stockpoints in the echelon its base stock level and allocations functions; for the end-stockpoints it prescribes their base stock levels. This policy is denoted by a pair $\left(y_{i}, \Psi_{i}\right)$, in which $\Psi_{i}$ summarizes the parameters downstream of stockpoint $i: \Psi_{i}=\emptyset$ if $i \in E$ and $\Psi_{i}=\cup_{j \in V_{i}}\left(z_{j}, y_{j}, \Psi_{j}\right)$ if $i \in M$.

Assign the following one-period costs (holding and penalty) to installation $i$ if its echelon stock at the beginning of the period is increased to $x$ :

$$
C_{i}(x)= \begin{cases}h_{i}\left(x-\mu_{i}\right), & i \in M \\ h_{i}\left(x-\mu_{i}\right)+\int_{x}^{\infty}\left(h_{i}+\sum_{j \in U_{i}} h_{j}+p_{i}\right)(u-x) \mathrm{d} F_{i}(u), & i \in E .\end{cases}
$$

The expected cost of echelon $i$ given the policy $\left(y_{i}, \Psi_{i}\right)$ is as follows.

Lemma 1 (Diks and De Kok, 1998) For replenishment policy $\left(y_{i}, \Psi_{i}\right)$ the average cost of echelon $i$ equals

$$
\begin{aligned}
D_{i}\left(y_{i}, \Psi_{i}\right)= & \int_{0}^{\infty} C_{i}\left(y_{i}-u\right) \mathrm{d} F_{i}^{\left(L_{i}\right)}(u)+\sum_{n \in V_{i}}\left(\int_{0}^{\Delta_{i}} D_{n}\left(y_{n}, \Psi_{n}\right) \mathrm{d} F_{i}^{\left(L_{i}\right)}(u)\right. \\
& \left.+\int_{\Delta_{i}}^{\infty} D_{n}\left(z_{n}\left(y_{i}-u\right), \Psi_{n}\right) \mathrm{d} F_{i}^{\left(L_{i}\right)}(u)\right) .
\end{aligned}
$$

Hence, the expected cost of echelon $i$ consists of the cost of stockpoint $i$ and the costs generated by its successors. These latter costs depend on whether or not stockpoint $i$ has sufficient stock to satisfy all the demand from its successors.

Under the balance assumption, Diks and De Kok (1998) show that the cost $D_{1}\left(y_{1}, \Psi_{1}\right)$ of the inventory system is minimized in the global optimal replenishment policy $\left(\hat{y}_{1}, \hat{\Psi}_{1}\right)$. This optimum can easily be implemented in a system under centralized control. But in practice, divergent systems often operate under decentralized control in which each stockpoint decides about its private base stock level $y_{i}$ and allocation functions $z_{j}, j \in V_{i}$. Once these decisions are made, they are implemented over an infinite horizon. Such a situation can be analysed by means of a (noncooperative) strategic game; see for example (Osborne and Rubinstein, 1994; Tijs, 2003). In such a game each player has to choose a strategy; this is done independently and simultaneously from the other players. Thereafter, each player incurs a cost that depends on the strategies chosen by all the players.

More formally, a strategic game consists of a set of players and for each player a strategy set and a cost function. Here, the player set is the set of stockpoints. Player $i$ 's strategy set $Y_{i}, i \in M$, is the set of all its feasible echelon base stock levels and allocation functions. For end-stockpoints $i \in E$ the strategy set $Y_{i}$ only contains the feasible echelon base stock levels. In the sequel, we also refer to a player's strategy as his policy. The average cost $H_{i}$ of player $i$ depends on the strategies $\left(y_{1}, \Psi_{1}\right)$ chosen by all players in the system and will be specified later. The goal of each player is to choose a strategy that minimizes its average cost. A solution of a strategic game is a Nash equilibrium. The strategies $\left(\bar{y}_{1}, \bar{\Psi}_{1}\right)$ are called a Nash equilibrium if individual deviations do not pay off: $H_{i}\left(\bar{y}_{1}, \bar{\Psi}_{1}\right) \leq H_{i}\left(\bar{y}_{1}^{-i}, \bar{\Psi}_{1}^{-i}\right)$, where the superscript $-i$ denotes that all parameters remain unchanged except player $i$ 's strategy.

\section{System under decentralized control}

Consider the divergent system under decentralized control, that is, each stockpoint decides upon its individual echelon base stock level and allocation functions. The true one-period 
$\operatorname{cost} \tilde{C}_{n}$ of stockpoint $n \in E$ equals holding costs for goods in stock and penalty costs for backlogs,

$$
\begin{aligned}
\tilde{C}_{n}(x) & =\int_{0}^{x}\left(h_{n}+\sum_{j \in U_{n}} h_{j}\right)(x-u) \mathrm{d} F_{n}(u)+\int_{x}^{\infty} p_{n}(u-x) \mathrm{d} F_{n}(u) \\
& =C_{n}(x)+\sum_{j \in U_{n}} h_{j}\left(x-\mu_{n}\right) .
\end{aligned}
$$

This implies that the true expected cost per period equals

$$
\begin{aligned}
\tilde{D}_{n}\left(y_{n}\right) & =\int_{0}^{\infty} \tilde{C}_{n}\left(y_{n}-u\right) \mathrm{d} F_{n}^{\left(L_{n}\right)}(u) \\
& =D_{n}\left(y_{n}\right)+\sum_{j \in U_{n}} h_{j}\left(y_{n}-\left(L_{n}+1\right) \mu_{n}\right) .
\end{aligned}
$$

Let $w_{n}$ denote the real base stock level as experienced by installation $n$. Then $w_{1}=y_{1}$ since the outside supplier always has ample stock to supply stockpoint 1 . Further, the demand of stockpoint $n$ is fulfilled if its supplier $i, i=\operatorname{pre}(n)$, has ample stock and otherwise he receives an allocated part of installation $i$ 's stock,

$$
w_{n}= \begin{cases}y_{n}, & \sum_{m \in V_{i}} y_{m} \leq w_{i}-u_{i}^{\left(L_{i}\right)}, \\ z_{n}\left(w_{i}-u_{i}^{\left(L_{i}\right)}\right), & \text { otherwise. }\end{cases}
$$

Occasionally we write $w_{n}\left(u_{i}^{\left(L_{i}\right)}\right)$ to stress the dependence of $w_{n}$ on $u_{i}^{\left(L_{i}\right)}$. Also, let $\mathbb{E}$ be the expectation. These notations allow for an easy formulation of the average cost of the system.

Lemma 2 The average cost of echelon 1 equals

$$
D_{1}\left(y_{1}, \Psi_{1}\right)=h_{1}\left(y_{1}-\left(L_{1}+1\right) \mu_{1}\right)+\sum_{i \in V_{1}} \mathbb{E} D_{i}\left(w_{i}, \Psi_{i}\right)
$$

where

$$
D_{i}\left(w_{i}, \Psi_{i}\right)=h_{i}\left(w_{i}-\left(L_{i}+1\right) \mu_{i}\right)+\sum_{n \in V_{i}} \mathbb{E} D_{n}\left(w_{n}\right)
$$

for $i \in W_{2}$ and

$$
\mathbb{E} D_{n}\left(w_{n}\right)=D_{n}\left(y_{n}\right)+\int_{\Delta_{i}}^{\infty}\left(D_{n}\left(z_{n}\left(w_{i}-u\right)\right)-D_{n}\left(y_{n}\right)\right) \mathrm{d} F_{i}^{\left(L_{i}\right)}(u)
$$

for $n \in E \cap V_{i}$.

The proof of this Lemma, as well as all subsequent proofs, may be found in the appendix.

The true expected cost $\tilde{H}_{n}$ per period for installation $n \in E$ is $\tilde{H}_{n}\left(y_{1}, \Psi_{1}\right)=\mathbb{E} \tilde{D}_{n}\left(w_{n}\right)$. For intermediate installations $i \in M$ the true expected cost equals inventory costs for goods in stock and in transit to installations $n \in V_{i}$.

$$
\begin{aligned}
\tilde{H}_{i}\left(y_{1}, \Psi_{1}\right)= & \mathbb{E} \int_{0}^{\infty}\left(h_{i}+\sum_{m \in U_{i}} h_{m}\right) \max \left(w_{i}-u-\sum_{n \in V_{i}} y_{n}, 0\right) \mathrm{d} F_{i}^{\left(L_{i}\right)}(u) \\
& +\sum_{n \in V_{i}} \int_{0}^{\infty}\left(h_{i}+\sum_{m \in U_{i}} h_{m}\right) u \mathrm{~d} F_{n}^{\left(L_{n}\right)}(u) .
\end{aligned}
$$

These cost functions reduce to simpler expressions, as shown below. 
Lemma 3 The true expected cost functions may be written as

$$
\tilde{H}_{i}\left(y_{1}, \Psi_{1}\right)=\left(h_{i}+\sum_{m \in U_{i}} h_{m}\right)\left(\mathbb{E} w_{i}-L_{i} \mu_{i}-\sum_{n \in V_{i}} \mathbb{E} w_{n}\right)+\sum_{n \in V_{i}}\left(h_{i}+\sum_{m \in U_{i}} h_{m}\right) L_{n} \mu_{n}
$$

for intermediate stockpoints $i \in M$, and

$$
\tilde{H}_{n}\left(y_{1}, \Psi_{1}\right)=\mathbb{E} D_{n}\left(w_{n}\right)+\sum_{j \in U_{n}} h_{j}\left(\mathbb{E} w_{n}-\left(L_{n}+1\right) \mu_{n}\right)
$$

for end-stockpoints $n \in E$.

From this we easily deduce the total cost per echelon, where $\tilde{H}_{e(i)}=\sum_{j \in e(i)} \tilde{H}_{j}$ is the cost of echelon $i$ resulting from cost function $\tilde{H}$.

Lemma 4 The total cost of echelon $i$ equals

$$
\tilde{H}_{e(i)}\left(y_{1}, \Psi_{1}\right)=\mathbb{E} D_{i}\left(w_{i}, \Psi_{i}\right)+\sum_{k \in U_{i}} h_{k}\left(\mathbb{E} w_{i}-\left(L_{i}+1\right) \mu_{i}\right) .
$$

As a corollary we obtain that the cost functions $\tilde{H}_{i}$ efficiently distribute the total cost of the inventory system among the stockpoints.

Corollary $5 \tilde{H}_{e(1)}\left(y_{1}, \Psi_{1}\right)=D_{1}\left(y_{1}, \Psi_{1}\right)$.

The cost functions $\tilde{H}_{i}$ may be interpreted as cost functions of a strategic game in which each stockpoint is a player. Upon doing so, each installation simultaneously and independently chooses a strategy - a pair consisting of a base stock level and a set of allocation functions — that minimizes its expected cost. These choices result in a Nash equilibrium of the game.

Theorem 6 A Nash equilibrium of the strategic game with cost functions $\tilde{H}_{i}$ is a policy $\left(y_{1}^{*}, \Psi_{1}^{*}\right)$ satisfying $y_{i}^{*}=\sum_{j \in V_{i}} y_{j}^{*}$ for $i \in M$, and $y_{n}^{*}$ minimizes $\tilde{H}_{n}\left(y_{1}, \Psi_{1}\right)$ for $n \in E$.

Notice that there exist multiple Nash equilibria because any set of allocation functions $\left\{z_{j}^{*}\right\}_{j \in V_{i}}$ is optimal. Further, no intermediate stockpoint will keep any extra stock in a Nash equilibrium $\left(y_{1}^{*}, \Psi_{1}^{*}\right)$, and its cost is $\tilde{H}_{i}\left(y_{1}^{*}, \Psi_{1}^{*}\right)=\sum_{n \in V_{i}}\left(h_{i}+\sum_{m \in U_{i}} h_{m}\right) L_{n} \mu_{n}$. Hence, the end-stockpoints $n \in E$ will incur large costs due to large probabilities of stock-out at their suppliers.

\section{Coordination mechanism}

In this section we introduce our mechanism for the three-stage divergent inventory system that results in: (i) a coordinated inventory system, and (ii) a fair allocation of the cost; all the installations enjoy cost savings compared to the situation under decentralized control. The mechanism consists of two rounds of negotiations between adjoining stages, namely first between the two most downstream stages and thereafter between the two most upstream stages. Finally, a feedback round will carry over the results from the second round of negotiations to the most downstream stage. 


\subsection{First round of negotiations}

Under decentralized control any end-stockpoint $n$ is confronted with large costs due to a high probability of stockouts for its supplier. Therefore - to reduce their costs - the endstockpoints will start negotiations with their immediate suppliers. During these negotiations we assume that the policies of the other stockpoints in the system remain unchanged; they remain equal to the policies as set under decentralized control.

For each stockpoint $i \in W_{2}$, the stockpoints $n \in V_{i}$ initiate negotiations with their supplier $i$. The installations $n \in V_{i}$ ask $i$ to increase its base stock level to some amount $y_{i}>\sum_{n \in V_{i}} y_{n}$. This results in a cost increase for stockpoint $i$ of size

$$
\tilde{H}_{i}\left(y_{1}, \Psi_{1}\right)-\tilde{H}_{i}\left(y_{1}^{*}, \Psi_{1}^{*}\right)=\left(h_{i}+h_{1}\right)\left(\mathbb{E} w_{i}-L_{i} \mu_{i}-\sum_{n \in V_{i}} \mathbb{E} w_{n}\right) .
$$

The installations in $V_{i}$ will compensate $i$ for this increase. In particular, installation $n \in V_{i}$ will pay the part

$$
\left(h_{i}+h_{1}\right)\left(\mathbb{E} z_{n}\left(w_{i}-u_{i}^{\left(L_{i}\right)}\right)-\mathbb{E} w_{n}\right) .
$$

These payments leave installation $i$ indifferent to cooperation since now its cost is equal to its initial cost. Hence, to persuade installation $i$ to participate, its new cost should be lower than the initial cost. To achieve this, installation $n$ uses its surplus - its cost savings minus the compensation paid to $i$ :

$$
\tilde{H}_{n}\left(y_{1}^{*}, \Psi_{1}^{*}\right)-\tilde{H}_{n}\left(y_{1}, \Psi_{1}\right)-\left(h_{i}+h_{1}\right)\left(\mathbb{E} z_{n}\left(w_{i}-u_{i}^{\left(L_{i}\right)}\right)-\mathbb{E} w_{n}\right) .
$$

Installation $i$ receives a fraction $\gamma_{i}$ of this surplus from all stockpoints $n$ it supplies.

The new cost for installation $n \in V_{i}$ resulting from these negotiations, includes its original $\operatorname{cost} \tilde{H}_{n}$, and the compensation and the fraction of its surplus paid to its supplier $i$.

$$
\begin{aligned}
H_{n}^{\prime}\left(y_{1}, \Psi_{1}\right)= & \tilde{H}_{n}\left(y_{1}, \Psi_{1}\right)+\left(h_{i}+h_{1}\right) \mathbb{E}\left(z_{n}\left(w_{i}-u_{i}^{\left(L_{i}\right)}\right)-w_{n}\right) \\
& +\gamma_{i}\left(\tilde{H}_{n}\left(y_{1}^{*}, \Psi_{1}^{*}\right)-\tilde{H}_{n}\left(y_{1}, \Psi_{1}\right)-\left(h_{i}+h_{1}\right) \mathbb{E}\left(z_{n}\left(w_{i}-u_{i}^{\left(L_{i}\right)}\right)-w_{n}\right)\right) \\
= & \left(1-\gamma_{i}\right)\left(\tilde{H}_{n}\left(y_{1}, \Psi_{1}\right)+\left(h_{i}+h_{1}\right) \mathbb{E}\left(z_{n}\left(w_{i}-u_{i}^{\left(L_{i}\right)}\right)-w_{n}\right)\right)+\gamma_{i} \tilde{H}_{n}\left(y_{1}^{*}, \Psi_{1}^{*}\right) .
\end{aligned}
$$

Along similar lines, the new cost for installation $i$ is its original cost $\tilde{H}_{i}$ reduced by the compensations and the fractions of the surplusses it receives of its buyers.

$$
\begin{aligned}
H_{i}^{\prime}\left(y_{1}, \Psi_{1}\right)= & \tilde{H}_{i}\left(y_{1}, \Psi_{1}\right)-\sum_{n \in V_{i}}\left(h_{i}+h_{1}\right) \mathbb{E}\left(z_{n}\left(w_{i}-u_{i}^{\left(L_{i}\right)}\right)-w_{n}\right) \\
& -\sum_{n \in V_{i}} \gamma_{i}\left(\tilde{H}_{n}\left(y_{1}^{*}, \Psi_{1}^{*}\right)-\tilde{H}_{n}\left(y_{1}, \Psi_{1}\right)-\left(h_{i}+h_{1}\right) \mathbb{E}\left(z_{n}\left(w_{i}-u_{i}^{\left(L_{i}\right)}\right)-w_{n}\right)\right) \\
= & \gamma_{i} \tilde{H}_{e(i)}\left(y_{1}, \Psi_{1}\right)-\gamma_{i} \tilde{H}_{e(i)}\left(y_{1}^{*}, \Psi_{1}^{*}\right)+\tilde{H}_{i}\left(y_{1}^{*}, \Psi_{1}^{*}\right) .
\end{aligned}
$$

Notice that these cost functions are a reallocation of the former ones:

$$
H_{e(i)}^{\prime}\left(y_{1}, \Psi_{1}\right)=\tilde{H}_{e(i)}\left(y_{1}, \Psi_{1}\right) ;
$$

the negotations conserve the cost of the echelon.

The outcome of this first round of negotiations is as follows. 
Theorem 7 In the first round of the negotiations the policy $\left(y_{1}^{a}, \Psi_{1}^{a}\right)$ is chosen by the installations. Installation $n \in V_{i}$ minimizes its cost $H_{n}^{\prime}\left(y_{1}, \Psi_{1}\right)$ in the global optimum, $y_{n}^{a}=\hat{y}_{n}$; installation $i$ minimizes its cost $H_{i}^{\prime}\left(y_{1}, \Psi_{1}\right)$ in $y_{i}^{a} \neq \hat{y}_{i}$ and $z_{n}^{a} \neq \hat{z}_{n}, n \in V_{i}$.

This policy is preferred by all parties involved in the negotiations.

Theorem 8 The negotiations in the first round reduce costs: $H_{j}^{\prime}\left(y_{1}^{a}, \Psi_{1}^{a}\right)<\tilde{H}_{j}\left(y_{1}^{*}, \Psi_{1}^{*}\right)$ for all installations $j$ in echelon $i$.

\subsection{Second round of negotiations}

After the first round of negotiations - between stockpoints $i \in W_{2}$ and their buyers - we proceed to the next round. In the three-stage model under consideration, $W_{3}=\{1\}$. Hence, in this round installation 1 and all installations $i \in V_{1}$ are negotiating. Each installation $i \in V_{1}$ participates on behalf of its echelon because the negotiations also have impact on the costs of the other installations in the echelon; among others, cost savings for installation $i$ result in lower compensations from installations $n \in V_{i}$ to $i$.

The second round of negotiations proceeds among similar lines as the first round. All installations $i \in V_{1}$ ask stockpoint 1 to increase its base stock level $y_{1}$ to some level $y_{1}>$ $\sum_{i \in V_{i}} y_{i}$. Recall that the policies of the other stockpoints remain unchanged: $y_{n}=y_{n}^{a}=\hat{y}_{n}$ for all $n \in V_{i}$ as determined by the previous round of negotiations. The cost increase for 1 equals

$$
\tilde{H}_{1}\left(y_{1}, \Psi_{1}\right)-\tilde{H}_{1}\left(y_{1}^{a}, \Psi_{1}^{a}\right)=h_{1}\left(y_{1}-L_{1} \mu_{1}-\sum_{i \in V_{1}} \omega_{i}\right) .
$$

Installation $i \in V_{1}$ will pay the compensation $h_{1} \mathbb{E}\left(z_{i}\left(y_{1}-u_{1}^{\left(L_{1}\right)}\right)-w_{i}\right)$. The surplus of echelon $i$ equals

$$
\tilde{H}_{e(i)}\left(y_{1}^{a}, \Psi_{1}^{a}\right)-\tilde{H}_{e(i)}\left(y_{1}, \Psi_{1}\right)-h_{1} \mathbb{E}\left(z_{i}\left(y_{1}-u_{1}^{\left(L_{1}\right)}\right)-w_{i}\right)
$$

due to equation (4). Installation 1 receives a fraction $\gamma_{1}$ of this surplus from any echelon $i \in V_{1}$. These payments result in new costs for echelon $i$ :

$$
\begin{aligned}
H_{e(i)}\left(y_{1}, \Psi_{1}\right)= & H_{e(i)}^{\prime}\left(y_{1}, \Psi_{1}\right)+h_{1} \mathbb{E}\left(z_{i}\left(y_{1}-u_{1}^{\left(L_{1}\right)}\right)-w_{i}\right) \\
& +\gamma_{1}\left(\tilde{H}_{e(i)}\left(y_{1}^{a}, \Psi_{1}^{a}\right)-\tilde{H}_{e(i)}\left(y_{1}, \Psi_{1}\right)-h_{1} \mathbb{E}\left(z_{i}\left(y_{1}-u_{1}^{\left(L_{1}\right)}\right)-w_{i}\right)\right) \\
= & \left(1-\gamma_{1}\right)\left(\tilde{H}_{e(i)}\left(y_{1}, \Psi_{1}\right)+h_{1} \mathbb{E}\left(z_{i}\left(y_{1}-u_{1}^{\left(L_{1}\right)}\right)-w_{i}\right)\right)+\gamma_{1} \tilde{H}_{e(i)}\left(y_{1}^{a}, \Psi_{1}^{a}\right) .
\end{aligned}
$$

The negotiations change the cost of stockpoint 1 to

$$
\begin{aligned}
H_{1}\left(y_{1}, \Psi_{1}\right)= & \tilde{H}_{1}\left(y_{1}, \Psi_{1}\right)-\sum_{i \in V_{1}} h_{1} \mathbb{E}\left(z_{i}\left(y_{1}-u_{1}^{\left(L_{1}\right)}\right)-w_{i}\right) \\
& -\sum_{i \in V_{1}} \gamma_{1}\left(\tilde{H}_{e(i)}\left(y_{1}^{a}, \Psi_{1}^{a}\right)-\tilde{H}_{e(i)}\left(y_{1}, \Psi_{1}\right)-h_{1} \mathbb{E}\left(z_{i}\left(y_{1}-u_{1}^{\left(L_{1}\right)}\right)-w_{i}\right)\right) \\
= & \gamma_{1} D_{1}\left(y_{1}, \Psi_{1}\right)+\tilde{H}_{1}\left(y_{1}^{a}, \Psi_{1}^{a}\right)-\gamma_{1} D_{1}\left(y_{1}^{a}, \Psi_{1}^{a}\right)
\end{aligned}
$$

The goal of each installation is to minimize its costs. This leads to the following result. 
Theorem 9 In the second round of negotiations the policy $\left(y_{1}^{b}, \Psi_{1}^{b}\right)$ is chosen by the installations. Echelon $i$ minimizes its cost $H_{e(i)}\left(y_{1}, \Psi_{1}\right)$ in $\left(y_{i}^{b}, \Psi_{i}^{b}\right)=\left(\hat{y}_{i}, \hat{\Psi}_{i}\right)$; stockpoint 1 minimizes its cost $H_{1}\left(y_{1}, \Psi_{1}\right)$ in $y_{1}^{b}=\hat{y}_{1}$ and $z_{i}^{b}=\hat{z}_{i}$ for all $i \in V_{1}$. Consequently, the global optimal policy is chosen: $\left(y_{1}^{b}, \Psi_{1}^{b}\right)=\left(\hat{y}_{1}, \hat{\Psi}_{1}\right)$.

We conclude from this theorem that after the second round of negotiations the system is coordinated because the global optimal policy $\left(\hat{y}_{1}, \hat{\Psi}_{1}\right)$ is selected. This outcome is preferred by all parties involved since they enjoy cost savings, as is shown in the next theorem.

Theorem 10 The negotiations in the second round reduce costs: $H_{1}\left(y_{1}^{b}, \Psi_{1}^{b}\right)=H_{1}\left(\hat{y}_{1}, \hat{\Psi}_{1}\right)<$ $\tilde{H}_{1}\left(y_{1}^{a}, \Psi_{1}^{a}\right)$ and $H_{e(i)}\left(y_{1}^{b}, \Psi_{1}^{b}\right)=H_{e(i)}\left(\hat{y}_{1}, \hat{\Psi}_{1}\right)<H_{e(i)}^{\prime}\left(y_{1}^{a}, \Psi_{1}^{a}\right)$ for all echelons $i \in V_{1}$.

\subsection{Feedback round}

Finally, the stockpoints $i \in W_{2}$ will inform their buyers about the outcome of the second round of negotiations. In this feedback round the cost $H_{e(i)}\left(\hat{y}_{1}, \hat{\Psi}_{1}\right)$ has to be divided among the installations in echelon $i$. Recall that

$$
\begin{aligned}
H_{e(i)}\left(\hat{y}_{1}, \hat{\Psi}_{1}\right)= & H_{i}^{\prime}\left(\hat{y}_{1}, \hat{\Psi}_{1}\right)+\sum_{n \in V_{i}} H_{n}^{\prime}\left(\hat{y}_{1}, \hat{\Psi}_{1}\right)+h_{1} \mathbb{E}\left(\hat{z}_{i}\left[\hat{y}_{1}-u_{1}^{\left(L_{1}\right)}\right]-w_{i}\right) \\
& +\gamma_{1}\left(\tilde{H}_{e(i)}\left(y_{1}^{a}, \Psi_{1}^{a}\right)-\tilde{H}_{e(i)}\left(\hat{y}_{1}, \hat{\Psi}_{1}\right)-h_{1} \mathbb{E}\left(\hat{z}_{i}\left[\hat{y}_{1}-u_{1}^{\left(L_{1}\right)}\right]-w_{i}\right)\right) .
\end{aligned}
$$

A natural division of this cost prescribes that stockpoint $n \in V_{i}$ pays $H_{n}^{\prime}\left(\hat{y}_{1}, \hat{\Psi}_{1}\right)$ and stockpoint $i$ pays the remaining

$$
H_{i}^{\prime}\left(\hat{y}_{1}, \hat{\Psi}_{1}\right)+\gamma_{1}\left(\tilde{H}_{e(i)}\left(y_{1}^{a}, \Psi_{1}^{a}\right)-\tilde{H}_{e(i)}\left(\hat{y}_{1}, \hat{\Psi}_{1}\right)-h_{1} \mathbb{E}\left(\hat{z}_{i}\left[\hat{y}_{1}-u_{1}^{\left(L_{1}\right)}\right]-w_{i}\right)\right) .
$$

This need not be unfavorable to $i$ because the change in policy from $\left(y_{1}^{a}, \Psi_{1}^{a}\right)$ to $\left(\hat{y}_{1}, \hat{\Psi}_{1}\right)$ results in an extra payment of $\gamma_{i}\left(H_{e(i)}\left(y_{1}^{a}, \Psi_{1}^{a}\right)-H_{e(i)}\left(\hat{y}_{1}, \hat{\Psi}_{1}\right)\right)$ from $n \in V_{i}$ to $i$. This covers the payments from $i$ to 1 if

$$
\gamma_{i}>\frac{H_{i}^{\prime}\left(\hat{y}_{1}, \hat{\Psi}_{1}\right)+\gamma_{1}\left(\tilde{H}_{e(i)}\left(y_{1}^{a}, \Psi_{1}^{a}\right)-\tilde{H}_{e(i)}\left(\hat{y}_{1}, \hat{\Psi}_{1}\right)-h_{1} \mathbb{E}\left(\hat{z}_{i}\left[\hat{y}_{1}-u_{1}^{\left(L_{1}\right)}\right]-w_{i}\right)\right)}{H_{e(i)}\left(y_{1}^{a}, \Psi_{1}^{a}\right)-H_{e(i)}\left(\hat{y}_{1}, \hat{\Psi}_{1}\right)}=: \underline{\gamma}_{i} .
$$

Notice that $0<\underline{\gamma}_{i}<1$. This immediately implies our main result:

Theorem 11 Consider the strategic game corresponding to the mechanism, in which the cost function of installation $i$ is $H_{i}$ for $i=1$ or $i \in V_{1} \cap W_{1}$, and $H_{i}^{\prime}$ otherwise. If $i \in V_{1}$ pays the compensation to 1 , and if $\gamma_{i}>\underline{\gamma}_{i}$ for $i \in W_{2}$ then the global optimal policy $\left(\hat{y}_{1}, \hat{\Psi}_{1}\right)$ is the unique Nash equilibrium of this game. Further, this Nash equilibrium is a fair cost allocation.

Hence, the mechanism coordinates the system because the global optimal replenishment policy is chosen by all the installations. Further, all stockpoints enjoy cost savings, compared to the initial situation under decentralized control, due to the fair cost allocation. 


\section{Conclusions}

In this paper a coordination mechanism for divergent multi-echelon inventory systems under periodic review and under decentralized control is presented and analysed. For ease of notation we studied a three-echelon system. Our coordination mechanism can readily be extended to general divergent $N$-echelon inventory systems. In that case, it will contain $N-1$ rounds of negotiations and $N-2$ rounds of feedback.

The decentralized control implies that the echelon base stock levels set by the installations need not be optimal from the perspective of the supply chain as a whole. The selfish installations act in their own self interest, which conflicts with global interests. A coordination mechanism is introduced to align the interests of the installations. The mechanism starts from the equilibrium outcome under decentralized control, which needs improvement since it is not globally optimal. In this outcome the most downstream firms incur the largest costs and therefore these will initiate negotiations with their supplier with the goal of decreasing their costs.

During the negotiations a mechanism is employed that is based upon two ideas: first, upstream installations should be fully compensated for cost increases due to larger base stock levels, and second, they should also receive a part of the surplus of the downstream installations. The mechanism changes the incentives in such a way that the global optimal policy is the unique Nash equilibrium of the corresponding strategic game. Hence, the system is coordinated. This result is due to the monetary side payments in the mechanism, which not only reduce the cost of the upstream installations but result in lower costs for all installations. Hence, the final cost allocation is a fair allocation. This is very important for the acceptance of the mechanism by all installations.

The coordination mechanism has several nice characteristics. First, the mechanism induces the upstream installation to carry inventory since the extra costs of this inventory will be reimbursed. Second, the monetary side payments redistribute the costs among the installations, so the cost of the system is conservated. Third, the mechanism is designed for implementation in systems with multiple independent firms, which are more complex than systems in which all firms belong to a single organisation. Finally, the resulting cost allocation is fair - all firms benefit from the mechanism.

\section{A Proofs}

\section{Proof of Lemma 2.}

Using lemma 1 and the definition of $C_{1}$, we derive

$$
\begin{aligned}
D_{1}\left(y_{1}, \Psi_{1}\right)= & \int_{0}^{\infty} h_{1}\left(y_{1}-u\right) \mathrm{d} F_{1}^{\left(L_{1}+1\right)}(u)+\sum_{i \in V_{1}}\left(\int_{0}^{\Delta_{1}} D_{i}\left(y_{i}, \Psi_{i}\right) \mathrm{d} F_{1}^{\left(L_{1}\right)}(u)\right. \\
& \left.+\int_{\Delta_{1}}^{\infty} D_{i}\left(z_{i}\left(y_{1}-u\right), \Psi_{i}\right) \mathrm{d} F_{1}^{\left(L_{1}\right)}(u)\right) \\
= & h_{1}\left(y_{1}-\left(L_{1}+1\right) \mu_{1}\right)+\sum_{i \in V_{1}} \mathbb{E} D_{i}\left(w_{i}, \Psi_{i}\right) .
\end{aligned}
$$


Along similar lines, stockpoints for $i \in W_{2}$

$$
\begin{aligned}
D_{i}\left(w_{i}, \Psi_{i}\right)= & \int_{0}^{\infty} h_{i}\left(w_{i}-u\right) \mathrm{d} F_{i}^{\left(L_{i}+1\right)}(u)+\sum_{n \in V_{i}}\left(\int_{0}^{w_{i}-\sum_{n \in V_{i}} y_{n}} D_{n}\left(y_{n}\right) \mathrm{d} F_{i}^{\left(L_{i}\right)}(u)\right. \\
& \left.+\int_{w_{i}-\sum_{n \in V_{i}} y_{n}}^{\infty} D_{n}\left(z_{n}\left(w_{i}-u\right)\right) \mathrm{d} F_{i}^{\left(L_{i}\right)}(u)\right) \\
= & h_{i}\left(w_{i}-\left(L_{i}+1\right) \mu_{i}\right)+\sum_{n \in V_{i}} \mathbb{E} D_{n}\left(w_{n}\right) .
\end{aligned}
$$

Finally,

$$
\begin{aligned}
& \mathbb{E} D_{n}\left(w_{n}\right) \\
& =\int_{0}^{w_{i}-\sum_{n \in V_{i}} y_{n}} D_{n}\left(y_{n}\right) \mathrm{d} F_{i}^{\left(L_{i}\right)}(u)+\int_{w_{i}-\sum_{n \in V_{i}} y_{n}}^{\infty} D_{n}\left(z_{n}\left(w_{i}-u\right)\right) \mathrm{d} F_{i}^{\left(L_{i}\right)}(u) \\
& =D_{n}\left(y_{n}\right)+\int_{w_{i}-\sum_{n \in V_{i}} y_{n}}^{\infty}\left(D_{n}\left(z_{n}\left(w_{i}-u\right)\right)-D_{n}\left(y_{n}\right)\right) \mathrm{d} F_{i}^{\left(L_{i}\right)}(u)
\end{aligned}
$$

for $n \in E \cap V_{i}$.

\section{Proof of Lemma 3.}

The true cost for stockpoint $n \in E, \tilde{H}_{n}\left(y_{1}, \Psi_{1}\right)=\mathbb{E} \tilde{D}_{n}\left(w_{n}\right)$, follows immediately from (1). For installation $i \in W_{2}$ we derive

$$
\begin{aligned}
\tilde{H}_{i}\left(y_{1}, \Psi_{1}\right)= & \mathbb{E} \int_{0}^{\infty}\left(h_{i}+\sum_{m \in U_{i}} h_{m}\right) \max \left(w_{i}-u-\sum_{n \in V_{i}} y_{n}, 0\right) \mathrm{d} F_{i}^{\left(L_{i}\right)}(u) \\
& +\sum_{n \in V_{i}} \int_{0}^{\infty}\left(h_{i}+\sum_{j \in U_{i}} h_{j}\right) u \mathrm{~d} F_{n}^{\left(L_{n}\right)}(u) \\
= & \mathbb{E} \int_{0}^{\infty}\left(h_{i}+h_{1}\right)\left(w_{i}-u-\sum_{n \in V_{i}} w_{n}\right) \mathrm{d} F_{i}^{\left(L_{i}\right)}(u) \\
& +\sum_{n \in V_{i}}\left(h_{i}+h_{1}\right) L_{n} \mu_{n} \\
= & \left(h_{i}+h_{1}\right)\left(\mathbb{E} w_{i}-L_{i} \mu_{i}-\sum_{n \in V_{i}} \mathbb{E} w_{n}\right)+\sum_{n \in V_{i}}\left(h_{i}+h_{1}\right) L_{n} \mu_{n},
\end{aligned}
$$

because $U_{i}=\{1\}$ for the three-echelon system under consideration. The cost $\tilde{H}_{1}\left(y_{1}, \Psi_{1}\right)$ for stockpoint 1 follows from $U_{1}=\emptyset$ and $\mathbb{E} w_{1}=y_{1}$.

Proof of Lemma 4.

This proof is by induction. The statement for $i \in W_{1}=E$ follows directly from lemma 3 . 
Now assume the statement holds for all $i \in W_{l}$. Let $m \in W_{l+1}$, then

$$
\begin{aligned}
\sum_{j \in e(m)} \tilde{H}_{j}\left(y_{1}, \Psi_{1}\right) & \\
= & \tilde{H}_{m}\left(y_{1}, \Psi_{1}\right)+\sum_{j \in V_{m}} \sum_{k \in e(j)} \tilde{H}_{k}\left(y_{k}, \Psi_{k}\right) \\
= & \left(h_{m}+\sum_{k \in U_{m}} h_{k}\right)\left(\mathbb{E} w_{m}-L_{m} \mu_{m}-\sum_{j \in V_{m}} \mathbb{E} w_{j}\right)+\sum_{j \in V_{m}}\left(h_{m}+\sum_{k \in U_{m}} h_{k}\right) L_{j} \mu_{j} \\
& +\sum_{j \in V_{m}}\left(\mathbb{E} D_{j}\left(w_{j}, \Psi_{j}\right)+\sum_{k \in U_{j}} h_{k}\left(\mathbb{E} w_{j}-\left(L_{j}+1\right) \mu_{j}\right)\right) \\
= & \left(h_{m}+\sum_{k \in U_{m}} h_{k}\right)\left(\mathbb{E} w_{m}-\left(L_{m}+1\right) \mu_{m}\right)+\sum_{j \in V_{m}} \mathbb{E} D_{j}\left(w_{j}, \Psi_{j}\right) \\
= & \mathbb{E} D_{m}\left(w_{m}, \Psi_{m}\right)+\sum_{k \in U_{m}} h_{k}\left(\mathbb{E} w_{m}-\left(L_{m}+1\right) \mu_{m}\right)
\end{aligned}
$$

where lemma 3 and the induction assumption are used in the second equality. The third equality uses $\sum_{k \in U_{j}} h_{k}=h_{m}+\sum_{k \in U_{m}} h_{k}, \sum_{j \in V_{m}} \mu_{j}=\mu_{m}$, and the final equality follows from lemma 2.

\section{Proof of Theorem 6.}

Stockpoint $i \in M$ has cost $\tilde{H}_{i}\left(y_{1}, \Psi_{1}\right)$ as specified in lemma 3. Notice that $\mathbb{E} w_{i}-L_{i} \mu_{i}-$ $\sum_{n \in V_{i}} \mathbb{E} w_{n} \geq 0$ because firms track echelon inventories, and $\sum_{n \in V_{i}}\left(h_{i}+\sum_{m \in U_{i}} h_{m}\right) L_{n} \mu_{n}$ are fixed costs. As a consequence minimal costs are reached if $y_{i}^{*}=\sum_{n \in V_{i}} y_{n}^{*}$ since then $\mathbb{E} w_{i}-L_{i} \mu_{i}-\sum_{n \in V_{i}} \mathbb{E} w_{n}=0$. Notice that this minimum does not depend on $z_{n}$.

Simultaneously, end-stockpoint $n$ minimizes its cost $\tilde{H}_{n}\left(y_{1}, \Psi_{1}\right)$. By lemma 3 , the first order condition for a minimum is

$$
\begin{aligned}
0 & =\frac{d}{d y_{n}} \mathbb{E} D_{n}\left(w_{n}\right)+h_{1} \frac{d}{d y_{n}} \mathbb{E} w_{n} \\
& =D_{n}^{\prime}\left(y_{n}\right) F_{1}^{\left(L_{1}\right)}\left(\Delta_{1}\right)+h_{1} F_{1}^{\left(L_{1}\right)}\left(\Delta_{1}\right) \\
& =\left(D_{n}^{\prime}\left(y_{n}\right)+h_{1}\right) F_{1}^{\left(L_{1}\right)}\left(\Delta_{1}\right),
\end{aligned}
$$

where $D_{n}^{\prime}\left(y_{n}\right)=\frac{d}{d y_{n}} D_{n}\left(y_{n}\right)$. Therefore $D_{n}^{\prime}\left(y_{n}\right)+h_{1}=0$ because $F_{1}^{\left(L_{1}\right)}\left(\Delta_{1}\right)$ is outside of the control of stockpoint $n$. By lemma 1,

$$
D_{n}^{\prime}\left(y_{n}\right)=\left(h_{n}+h_{1}+p_{n}\right) F_{n}^{\left(L_{n}+1\right)}\left(y_{n}\right)-h_{1}-p_{n},
$$

so end-stockpoint $n$ selects the base stock level $y_{n}^{*}$ satisfying $F_{n}^{\left(L_{n}+1\right)}\left(y_{n}^{*}\right)=p_{n} /\left(h_{n}+h_{1}+p_{n}\right)$. One easily checks that the second order condition for a minimum is satisfied.

\section{Proof of Theorem 7.}

Minimizing $H_{n}^{\prime}\left(y_{1}, \Psi_{1}\right)$ with respect to $y_{n}$ is equivalent to minimizing

$$
\tilde{H}_{n}\left(y_{1}, \Psi_{1}\right)+\left(h_{i}+h_{1}\right) \mathbb{E}\left(z_{n}\left(w_{i}-u_{i}^{\left(L_{i}\right)}\right)-w_{n}\right)
$$


because the other terms in $H_{n}^{\prime}$ are constant. Then

$$
\begin{aligned}
& \tilde{H}_{n}\left(y_{1}, \Psi_{1}\right)+\left(h_{i}+h_{1}\right) \mathbb{E}\left(z_{n}\left(w_{i}-u_{i}^{\left(L_{i}\right)}\right)-w_{n}\right) \\
& \quad=\mathbb{E} D_{n}\left(w_{n}\right)+\left(\sum_{j \in U_{n}} h_{j}\right)\left(\omega_{n}-\left(L_{n}+1\right) \mu_{n}\right)+\left(h_{i}+h_{1}\right) \mathbb{E}\left(z_{n}\left(w_{i}-u_{i}^{\left(L_{i}\right)}\right)-w_{n}\right) \\
& \quad=\mathbb{E} D_{n}\left(w_{n}\right)+\left(h_{i}+h_{1}\right)\left(\mathbb{E} z_{n}\left(w_{i}-u_{i}^{\left(L_{i}\right)}\right)-\left(L_{n}+1\right) \mu_{n}\right),
\end{aligned}
$$

so, minimizing $H_{n}^{\prime}\left(y_{1}, \Psi_{1}\right)$ with respect to $y_{n}$ boils down to minimizing $\mathbb{E} D_{n}\left(w_{n}\right)$. Using lemma 2 , the first order condition for a minimum is

$$
\begin{aligned}
0 & =\frac{d}{d y_{n}} D_{n}\left(y_{n}\right)+\left(D_{n}\left(z_{n}\left(\sum_{n \in V_{i}} y_{n}\right)\right)-D_{n}\left(y_{n}\right)\right) f_{i}^{\left(L_{i}\right)}\left(\Delta_{i}\right)-\int_{\Delta_{i}}^{\infty} \frac{d}{d y_{n}} D_{n}\left(y_{n}\right) \mathrm{d} F_{i}^{\left(L_{i}\right)}(u) \\
& =\left(\frac{d}{d y_{n}} D_{n}\left(y_{n}\right)\right) F_{i}^{\left(L_{i}\right)}\left(\Delta_{i}\right) .
\end{aligned}
$$

Thus $\frac{d}{d y_{n}} D_{n}\left(y_{n}\right)=0$, since $\Delta_{i}>0$ by assumption. By lemma 1 this equivalent to minimizing $D_{n}\left(y_{n}\right)$, the allocated cost for installation $n$ under centralized control. Hence, stockpoint $n$ chooses $y_{n}=\hat{y}_{n}$ - the global optimum is reached. One easily checks that the second order condition is satisfied.

According to (3) and lemma 4, minimizing $H_{i}^{\prime}\left(y_{1}, \Psi_{1}\right)$ is equivalent to minimizing

$$
\tilde{H}_{e(i)}\left(y_{1}, \Psi_{1}\right)=\mathbb{E} D_{i}\left(w_{i}^{*}, \Psi_{i}\right)+h_{1}\left(\mathbb{E} w_{i}^{*}-\left(L_{i}+1\right) \mu_{i}\right),
$$

where $w_{i}^{*}=z_{i}\left(y_{1}^{*}-u_{1}^{\left(L_{1}\right)}\right)$. Because the last term on the right hand side is constant, we have to minimize $\mathbb{E} D_{i}\left(w_{i}^{*}, \Psi_{i}\right)$. Comparing this to the cost $D_{i}\left(y_{i}, \Psi_{i}\right)$ of echelon $i$ under centralized control as denoted in lemma 1 , we see that the holding costs $h_{i}$ are changed to $h_{i}+h_{1}$, the lead time is increased from $L_{i}$ to $L_{i}+L_{1}$ and its base stock level is $w_{i}^{*}$ instead of $y_{i}$. Due to these changes, the minimum differs from the minimum in the global optimum: $y_{i}^{a} \neq \hat{y}_{i}$ and $z_{n}^{a} \neq \hat{z}_{n}$ for all $n \in V_{i}$.

\section{Proof of Theorem 8.}

For stockpoints $n \in V_{i}$

$$
\begin{aligned}
H_{n}^{\prime}\left(y_{1}^{a}, \Psi_{1}^{a}\right) & <H_{n}^{\prime}\left(y_{1}^{*}, \Psi_{1}^{*}\right) \\
& =\left(1-\gamma_{i}\right)\left(\tilde{H}_{n}\left(y_{1}^{*}, \Psi_{1}^{*}\right)+\left(h_{i}+h_{1}\right) \mathbb{E}\left(z_{n}\left(w_{i}^{*}-u_{i}^{\left(L_{i}\right)}\right)-w_{n}^{*}\right)\right)+\gamma_{i} \tilde{H}_{n}\left(y_{1}^{*}, \Psi_{1}^{*}\right) \\
& =\tilde{H}_{n}\left(y_{1}^{*}, \Psi_{1}^{*}\right),
\end{aligned}
$$

where the inequality is due to theorem 7 , and the first equality follows from (2). Also, $w_{j}^{*}$ is the real base stock level $w_{j}$ depending on policy $\left(y_{1}^{*}, \Psi_{1}^{*}\right)$, which implies $z_{n}\left(w_{i}^{*}-u_{i}^{\left(L_{i}\right)}\right)-w_{n}^{*}=0$. Further,

$$
\begin{aligned}
H_{i}^{\prime}\left(y_{1}^{a}, \Psi_{1}^{a}\right) & <H_{n}^{\prime}\left(y_{1}^{*}, \Psi_{1}^{*}\right) \\
& =\gamma_{i} \tilde{H}_{e(i)}\left(y_{1}^{*}, \Psi_{1}^{*}\right)-\gamma_{i} \tilde{H}_{e(i)}\left(y_{1}^{*}, \Psi_{1}^{*}\right)+\tilde{H}_{i}\left(y_{1}^{*}, \Psi_{1}^{*}\right) \\
& =\tilde{H}_{i}\left(y_{1}^{*}, \Psi_{1}^{*}\right),
\end{aligned}
$$

where theorem 7 and (3) are used. 


\section{Proof of Theorem 9.}

Recall that $y_{n}=\hat{y}_{n}$ for $n \in V_{i}$. Using (5) and lemma 4, we obtain

$$
\begin{aligned}
H_{e(i)}\left(y_{1}, \Psi_{1}\right)= & \left(1-\gamma_{1}\right)\left(\tilde{H}_{e(i)}\left(y_{1}, \Psi_{1}\right)+h_{1} \mathbb{E}\left(z_{i}\left(y_{1}-u_{1}^{\left(L_{1}\right)}\right)-w_{i}\right)\right)+\gamma_{1} \tilde{H}_{e(i)}\left(y_{1}^{a}, \Psi_{1}^{a}\right) \\
= & \left(1-\gamma_{1}\right)\left(\mathbb{E} D_{i}\left(w_{i}, \Psi_{i}\right)+h_{1}\left(\mathbb{E} w_{i}-\left(L_{i}+1\right) \mu_{i}\right)+h_{1} \mathbb{E}\left(z_{i}\left(y_{1}-u_{1}^{\left(L_{1}\right)}\right)-w_{i}\right)\right) \\
& +\gamma_{1} \tilde{H}_{e(i)}\left(y_{1}^{a}, \Psi_{1}^{a}\right) \\
= & \left(1-\gamma_{1}\right)\left(\mathbb{E} D_{i}\left(w_{i}, \Psi_{i}\right)+h_{1}\left(\mathbb{E} z_{i}\left(y_{1}-u_{1}^{\left(L_{1}\right)}\right)-\left(L_{i}+1\right) \mu_{i}\right)\right) \\
& +\gamma_{1} \tilde{H}_{e(i)}\left(y_{1}^{a}, \Psi_{1}^{a}\right) .
\end{aligned}
$$

Therefore, minimizing $H_{e(i)}\left(y_{1}, \Psi_{1}\right)$ is equivalent to minimizing

$$
\begin{aligned}
\mathbb{E} D_{i}\left(w_{i}, \Psi_{i}\right)= & h_{i}\left(\mathbb{E} w_{i}-\left(L_{i}+1\right) \mu_{i}\right) \\
& +\mathbb{E} \sum_{n \in V_{i}}\left(\int_{0}^{w_{i}-\sum_{m \in V_{i}} y_{m}} D_{n}\left(y_{n}\right) \mathrm{d} F_{i}^{\left(L_{i}\right)}(u)\right. \\
& \left.+\int_{w_{i}-\sum_{m \in V_{i}} y_{m}}^{\infty} D_{n}\left(z_{n}\left(w_{i}-u\right)\right) \mathrm{d} F_{i}^{\left(L_{i}\right)}\right) .
\end{aligned}
$$

We compare this to the allocated cost for echelon $i$ under centralized control (see lemma 1),

$$
\begin{aligned}
D_{i}\left(y_{i}, \Psi_{i}\right)= & h_{i}\left(y_{i}-\left(L_{i}+1\right) \mu_{i}\right) \\
& +\sum_{n \in V_{i}}\left(\int_{0}^{\Delta_{i}} D_{n}\left(y_{n}\right) \mathrm{d} F_{i}^{\left(L_{i}\right)}(u)\right. \\
& \left.+\int_{\Delta_{i}}^{\infty} D_{n}\left(z_{n}\left(y_{i}-u\right)\right) \mathrm{d} F_{i}^{\left(L_{i}\right)}\right) .
\end{aligned}
$$

From this comparison we conclude that minimizing $\mathbb{E} D_{i}\left(w_{i}, \Psi_{i}\right)$ with respect to the allocation functions $\left(z_{n}\right)_{n \in V_{i}}$ results in the same outcome as minimizing $D_{i}\left(y_{i}, \Psi_{i}\right)$, namely $z_{n}=\hat{z}_{n}$.

Also, the function $D_{i}\left(y_{i}, \hat{\Psi}_{i}\right)$ is convex in $y_{i}$ with minimum in $y_{i}=\hat{y}_{i}$, and $w_{i}$ is a continuous piecewise function in $y_{i}$ that is increasing (Diks and De Kok, 1998). Hence, $\mathbb{E} D_{i}\left(w_{i}, \Psi_{i}\right)$ is minimized in $y_{i}=\hat{y}_{i}$.

By (6), minimizing $H_{1}\left(y_{1}, \Psi_{1}\right)$ is equivalent to minimizing $D_{1}\left(y_{1}, \Psi_{1}\right)$ - the total cost of the inventory system. Hence, stockpoint 1 chooses the same policy as in the global optimum.

\section{Proof of Theorem 10.}

For echelon $i \in V_{1}$

$$
\begin{aligned}
H_{e(i)}\left(\hat{y}_{1}, \hat{\Psi}_{1}\right) & <H_{e(i)}\left(y_{1}^{a}, \Psi_{1}^{a}\right) \\
& =\left(1-\gamma_{1}\right)\left(\tilde{H}_{e(i)}\left(y_{1}^{a}, \Psi_{1}^{a}\right)+h_{1} \mathbb{E}\left(z_{i}\left(y_{1}^{a}-u_{1}^{\left(L_{1}\right)}\right)-w_{i}\right)\right)+\gamma_{1} \tilde{H}_{e(i)}\left(y_{1}^{a}, \Psi_{1}^{a}\right) \\
& =\tilde{H}_{e(i)}\left(y_{1}^{a}, \Psi_{1}^{a}\right)
\end{aligned}
$$

because of theorem 9 and $z_{i}\left(y_{1}^{a}-u_{1}^{\left(L_{1}\right)}\right)=w_{i}$. Along similar lines, we deduce

$$
\begin{aligned}
H_{1}\left(\hat{y}_{1}, \hat{\Psi}_{1}\right) & <H_{1}\left(y_{1}^{a}, \Psi_{1}^{a}\right) \\
& =\gamma_{1} D_{1}\left(y_{1}^{a}, \Psi_{1}^{a}\right)+\tilde{H}_{1}\left(y_{1}^{a}, \Psi_{1}^{a}\right)-\gamma_{1} D_{1}\left(y_{1}^{a}, \Psi_{1}^{a}\right) \\
& =\tilde{H}_{1}\left(y_{1}^{a}, \Psi_{1}^{a}\right) ;
\end{aligned}
$$


stockpoint 1 also saves costs.

\section{References}

[1] Arshinder, A Kanda, Deshmukh SG. Supply chain coordination: Perspectives, empirical studies and research directions. International Journal of Production Economics 2008; $115 ; 316-335$.

[2] Cachon GP. Stock wars: inventory competition in a two-echelon supply chain with multiple retailers. Operations Research 2001; 49; 658-647.

[3] Diks EB, De Kok AG. Optimal control of a divergent multi-echelon inventory system. European Journal of Operational Research 1998; 111; 75-97.

[4] Lee H, Whang S. Decentralized multi-echelon supply chains: incentives and information. Management Science 1999; 45; 633-640.

[5] Leng M, Zhu A. Side-payment contracts in two-person nonzero-sum supply chain games: Review, discussion and applications. European Journal of Operational Research 2009; $196 ; 600-618$.

[6] Li X, Wang Q. Coordination mechanisms of supply chain systems. European Journal of Operational Research 2007; 179; 1-16.

[7] Osborne MJ, Rubinstein A. A course in game theory. MIT Press: Cambridge; 1994.

[8] Seliaman ME, Ahmad AR. Optimizing inventory decisions in a multi-stage supply chain under stochastic demand. Applied Mathematics and Computation 2008; 206; 538-542.

[9] Tijs S. Introduction to game theory. Hindustan Book Agency: New Delhi; 2003.

[10] Zijm WHM, Timmer J. Coordination mechanisms for inventory control in three-echelon serial and distribution systems. Annals of Operations Research 2008; 158; 161-182. 